\title{
Additives for reducing the toxicity of respirable crystalline silica. SILIFE project
}

\author{
Eliseo Monfort ${ }^{1,}$, Ana López-Lilao ${ }^{1}$, Alberto Escrig ${ }^{1}$, Maria Jesus Ibáñez ${ }^{1}$, Guliana Bonvicini ${ }^{2}$, Otto Creutzenberg ${ }^{3}$, and \\ Christina Ziemann ${ }^{3}$ \\ ${ }^{1}$ Instituto de Tecnología Cerámica, Universitat Jaume I, Castellón, Spain \\ ${ }^{2}$ Centro Ceramico di Bologna (CCB), Bologna, Italy \\ ${ }^{3}$ Fraunhofer Institute for Toxicology and Experimental Medicine (ITEM), Hannover, German
}

\begin{abstract}
Prolonged inhalation of crystalline silica particles has long been known to cause lung inflammation and development of the granulomatous and a fibrogenic lung disease known as silicosis. The International Agency for Research on Cancer (IARC) has classified Respirable Crystalline Silica (RCS) in the form of quartz and cristobalite from occupational sources as carcinogenic for humans (category 1). In this regard, numerous studies suggest that the toxicity of quartz is conditioned by the surface chemistry of the quartz particles and by the density and abundance of silanol groups. Blocking these groups to avoid their interaction with cellular membranes would theoretically be possible in order to reduce or even to eliminate the toxic effect. In this regard, the main contribution of the presented research is the development of detoxifying processes based on coating technologies at industrial scale, since the previous studies reported on literature were carried out at lab scale. The results obtained in two European projects showed that the wet method to obtain quartz surface coatings (SILICOAT project) allows a good efficiency in inhibiting the silica toxicity, and the preliminary results obtained in an ongoing project (SILIFE) suggest that the developed dry method to coat quartz surface is also very promising. The development of both coating technologies (wet and a dry) should allow these coating technologies to be applied to a high variety of industrial activities in which quartz is processed. For this reason, a lot of end-users of quartz powders will be potentially benefited from a reduced risk associated to the exposure to RCS.
\end{abstract}

\section{Introduction}

Total European usage of crystalline silica (i.e. quartz and cristobalite) is measured in thousands of millions of tonnes per annum. It is used in many manufacturing industries [1-3] such as quarrying, mining, mineral processing (e.g. drying, grinding, bagging, and handling [4]); slate working [5], stone crushing and dressing; foundry work; brick and ceramic tile making; some ceramic refractory processes; construction work [6-7], including work with stone [8], concrete, brick and some insulation boards; tunnelling; building restoration; and the pottery and ceramic industries [9-12]. Hence, a vast number of European workers (4 million [13]) are potentially exposed to crystalline silica dust at their workplace.

In this regard, it is long established that prolonged inhalation of crystalline silica particles causes lung inflammation and development of the granulomatous and fibrogenic lung disease known as silicosis. Furthermore, the International Agency for Research on Cancer (IARC) classified Respirable Crystalline Silica (RCS) in the form of quartz and cristobalite from occupational sources as carcinogenic for humans (category 1, [14]). In this regard, numerous studies suggest that the toxicity of quartz is conditioned by the surface chemistry of the quartz particles and, in particular, by the density and abundance of silanol groups [15].

Blocking these groups to avoid the interaction with cellular membranes would theoretically be possible in order to reduce or even to eliminate the toxic effect. This postulate has been repeatedly verified by various researchers [15-23] who managed to reduce its toxicity at lab scale, on treating quartz with different substances: polyvinylpyridine-N-oxide (PVPNO) [15], organosilanes [16-17], aluminium lactate [18- 22] and strong Lewis acids, such as $\mathrm{AlCl}_{3}$ or $\mathrm{FeCl}_{3}$ [23].

The mechanism by which these substances act consists of the formation of a surface coating on the quartz so that its silanol groups are no longer accessible for interaction in biological media. Fig. 1 shows an example of this process using propyltrimetoxysilane (PTMO) as coating agent, in which two consecutive chemical reactions take place: 1) hydrolysis of one methoxy group of PTMO releasing methanol as coreaction product; and 2) silanization of quartz, in this stage the hydrolysed PTMO reacts with the silanol groups of quartz to form a very stable covalent bond.

Bearing in mind this background, this study was therefore undertaken to develop coating application

\footnotetext{
Corresponding author: eliseo.monfort@itc.uji.es
} 
processes at industrial scale to reduce the RCS toxicity. Regarding this fact, by the integration of these coating processes into the quartz producer manufacturing process, a great number of end user of quartz powders may potentially be benefit from a reduced risk associated to the exposure to RCS.
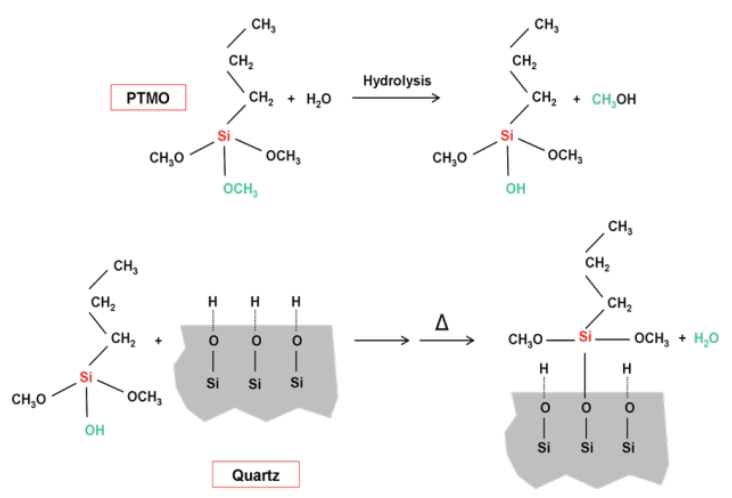

Fig. 1. Hydr.olysis and silanization reactions.

\section{Materials and methodology}

\subsection{Materials}

Although a wide range of compounds were studied, for the sake of brevity only the results obtained with SIVO160, the additive that exhibited the most promising behaviour, are presented. SIVO160 is a commercial aminosilane polymer used for coating siliceous as well as metallic surfaces.

An industrial quartz, used in a sanitary ware composition, was selected. Its specific surface area was $0.87 \mathrm{~m}^{2} \mathrm{~g}^{-1}$ and its particle size distribution (PSD) is shown in Fig. 2.

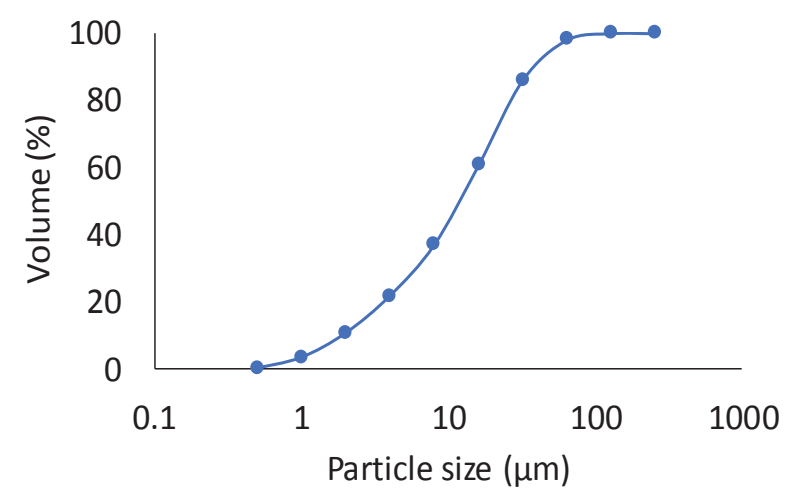

Fig. 2. PSD of the selected quartz.

\subsection{Methodology}

The followed methodology can be divided into two blocks: quartz coating and coating effectiveness assessment.

\subsubsection{Quartzes coating}

\subsubsection{Wet method (SILICOAT)}

1. Dispersion of the additive in water. The additive was incorporated into the quantity of water required in the formula. Certain organosilanes required preliminary hydrolysis, as hydrolysis took place more quickly in acid medium, this preliminary step was performed in the relevant cases, adding a certain quantity of $\mathrm{HCl} 0.01 \mathrm{M}$ to the additive and stirring until a homogeneous liquid was obtained (Fig. 3, left).

3. Addition of the quartz. The resulting suspension was stirred for the corresponding reaction time (Fig. 3, right).

4. Filtering the suspension and washing with ethanol. Once the reaction time had ended, the suspension was filtered with a Büchner flask and the resulting powder was repeatedly washed with ethanol to remove any surplus additive.

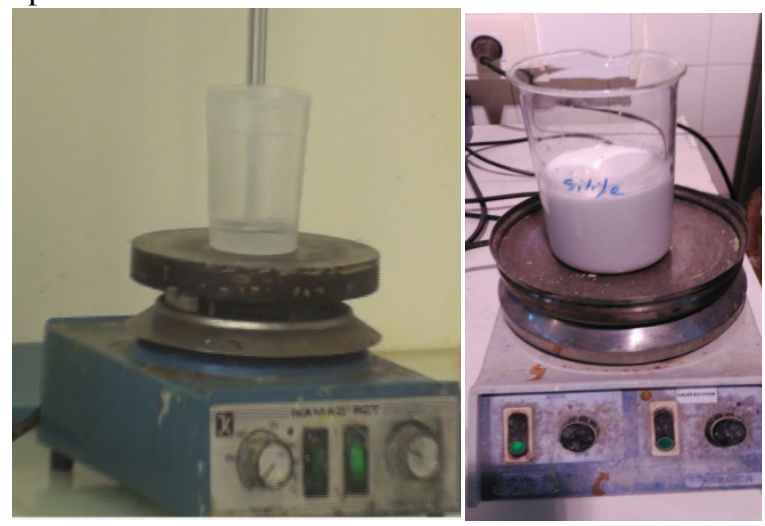

Fig. 3. Hydrolysis with $\mathrm{HCl}$ (left). Stirring suspension with quartz (right).

\subsubsection{Dry method (SILIFE)}

The coating has been performed using a high shear-rate plow mixer LÖDIGE M5R with a capacity up to 41 . The silane feeding has been carried out by an injector feeder (Fig. 4).
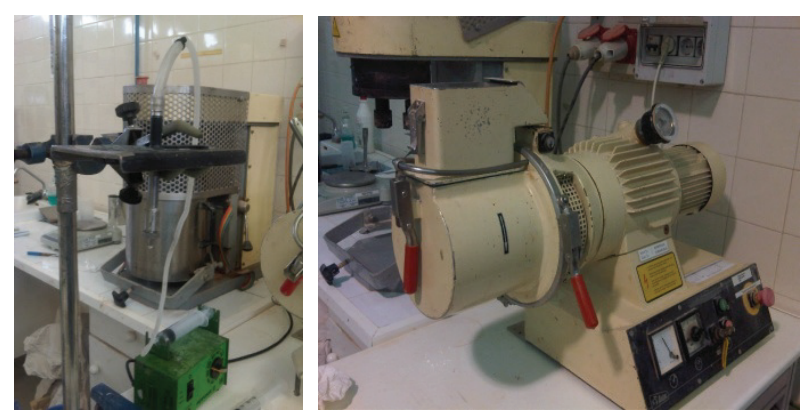

Fig. 4. Silane feeding (left) and mixer (right).

As in the wet method, after this process, the obtained quartz is rinsed with ethanol to remove any excess of silane and the obtained samples is dried with infrared lamps. 


\subsubsection{Coating effectiveness assessment}

Zeta potential ( $\varsigma$ potential) is a measure of the electrical charge in the surface of the suspended particles. It is thus related with the density of silanol groups in the quartz particles surface. As a consequence, the addition of the coating agent to quartz suspensions should increase or reduce the $\varsigma$ potential of the quartz (which is negative at $\mathrm{pH}>2$ ) and coating effectiveness can be measured in this manner. In this sense, it should be highlighted that some authors pointed out that the maximum inhibition of quartz toxicity is produced by rendering it iso-electric [24]. Moreover, the measurement of $\zeta$ potential was found to be extremely sensitive (modifications in surface charge due to the addition of less than $0.05 \% \mathrm{w} / \mathrm{w}$ of aminosilanes can be perfectly detected) and $\zeta$ potential is well correlated with toxicological results.

\section{Results and discussion}

The obtained results are divided into three blocks: wet method application results and validation of dry method.

\subsection{Coating effectiveness}

Thee variation of zeta potential with the proportioned quantity is represented in Fig. 5. From this figure, it can be observed that iso-electric point was associated with approximately $0.2 \%$ of SIVO160.

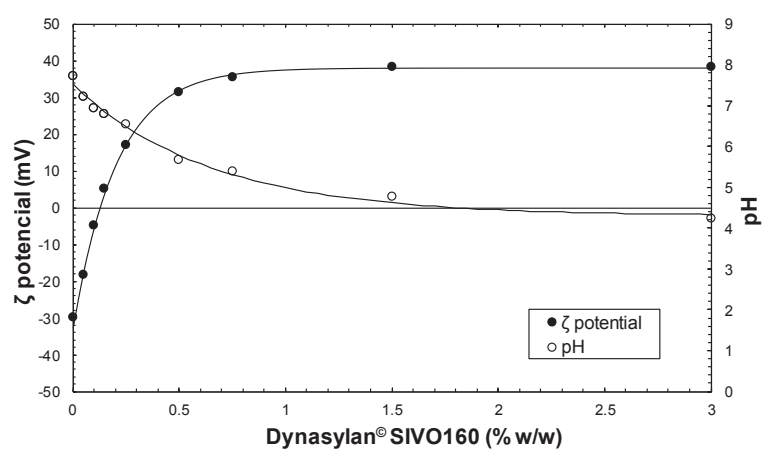

Fig. 5. Variation of zeta potential.

The most promising coating treatments at different concentrations were tested for toxicity. Fig. 6 shows the in vitro determination results of coating effectiveness. In these tests $\mathrm{Al}_{2} \mathrm{O}_{3}$ was used as particulate negative (PNC) and DQ12 as quartz positive control, this quartz is widely used in the literature as positive control, therefore the results may be compared with other studies on RCS toxicity. The toxicological tests presented in Fig. 6 are LDH release (Fig. 6, top) and the tail intensity in the alkaline comet assay (Fig. 6, bottom), which were used to detect membrane and direct DNA damage, respectively.

The in vitro toxicological results evidenced that the oligomeric, amino-modified siloxane SIVO160 seemed to possess considerable quartz toxicity-quenching potential. The toxicological study of the treated quartzes was completed in the project with in vivo tests, a more comprehensive information can be found in a specific paper [24].
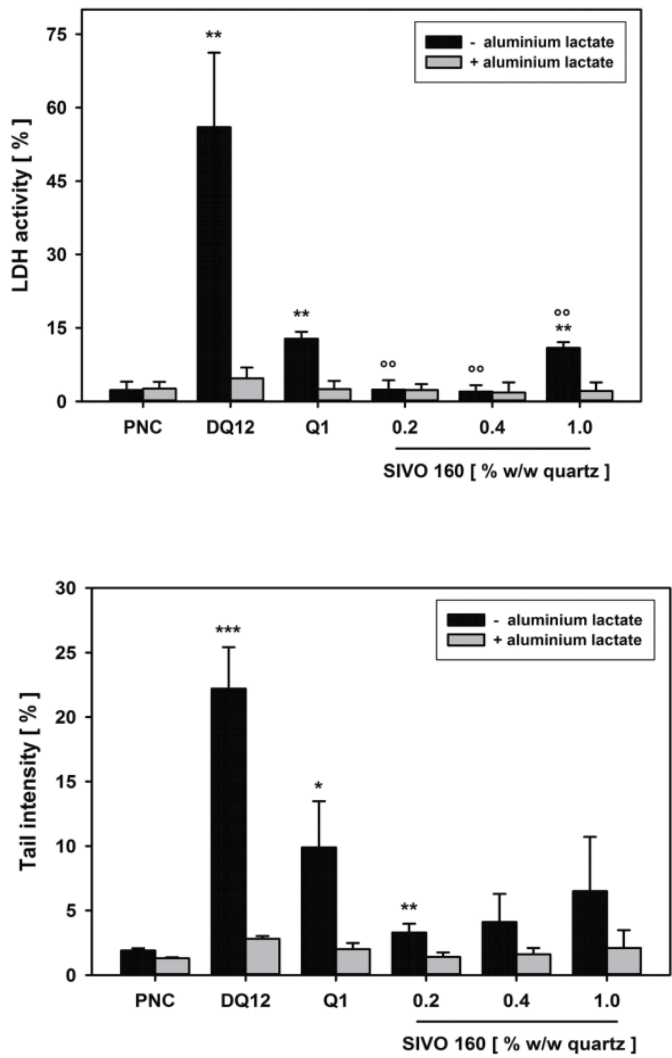

Fig. 6. Toxicological tests. Data represent means \pm SD of three independent experiments.

$* / * * * * *$ Significantly different from PNC: $\mathrm{P} \leq 0.05 / 0.01 / 0.001$, respectively, Student's t-test for unpaired values, two tailed. $0 / 00 / 000$ Significantly different from uncoated Q1: P $\leq$ $0.05 / 0.01 / 0.001$, respectively, Student's t-test for paired values, two tailed.

\subsection{Dry method versus wet method}

By the wet method, the selected treatments provided very satisfactory results in terms of toxicity, but unfortunately a lot of industrial processes using quartz do not include a wet stage, therefore in order to allow this coating technology to be implemented of in a wide variety of industrial applications, a new European project was proposed. Hence, the aim of the SILIFE project is to develop a dry method, based in the process proposed in SILICOAT, for reducing RCS toxicity at industrial scale. According to the surveyed literature, the use of a dry application method to this aim has not been previously reported.

Fig. 7 shows the preliminary results obtained so far applying this methodology and the comparison with those obtained by the wet application method (previous study).

The lab outcomes reported in Fig. 7 suggest that, surprisingly, from the physicochemical viewpoint, the dry method may be developed using similar dosages to those employed in the wet method. If these results are verified at industrial scale (nowadays under 
development) and by the toxicological assays, it would be a very promising technology.

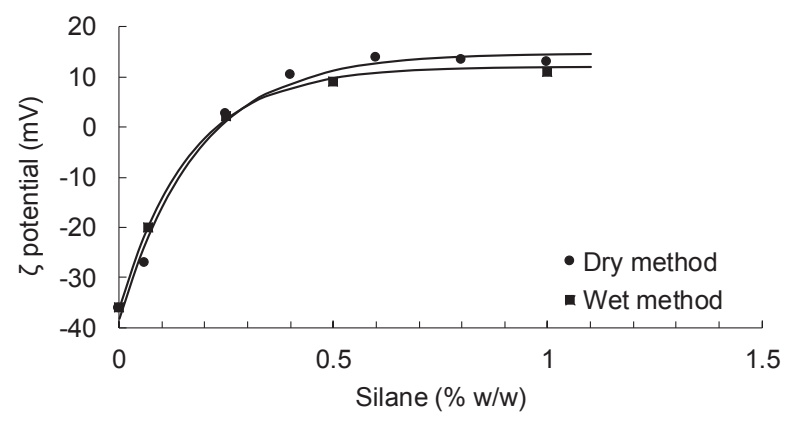

Fig. 7. Dry method versus wet method.

\section{Conclusions}

The results of a previous project (SILICOAT) showed that the wet method to industrially obtain quartz surface coatings allows a good efficiency in inhibiting the toxicological effect of silanol groups (verified by means of "in vitro" and "in vivo" tests) at relatively low doses. The developed process may be readily implemented at industrial scale, provided that they include a wet stage.

The lab results obtained in the SILIFE ongoing project suggest that the developed dry method to coat quartz surface is also very promising. Nevertheless, these preliminary physicochemical results must be scaled up and tested from the toxicological point of view.

The development of both coating technologies (wet and a dry) should allow these coating technologies to be applied at industrial scale in a high variety of industrial activities in which quartz is processed. For this reason, a lot of end-users of quartz powders will be potentially benefited from a reduced risk associated to the exposure to RCS.

\section{Acknowledgements}

This study was part of the SILICOAT project (FP7/2007-2013) and SILIFE project (LIFE14 ENV/ES/00238).

\section{References}

1. European Network for Silica (NEPSI): Good Practice Guide on Workers Health Protection through the Good Handling and Use of Crystalline Silica and Products Containing It, (2011). Available from: www.nepsi.eu (accessed April, 2015)

2. European Network for Silica (NEPSI): Agreement on Workers' Health Protection Through the Good Handling and Use of Crystalline Silicas and Products Containing It, (2006). Available from: www.nepsi.eu (accessed April, 2015)

3. D.L. Radnoff, M.K. Kutz, Ann. Occup. Hyg. 58, 19-27 (2014)
4. M.H. Fulekar, Ann. Occup. Hyg. 43, 269-73 (1999)

5. BE, Bang, H. Suhr, Ann. Occup. Hyg. 42, 557-63 (1998)

6. M.E Lumens, T. Spee, Ann. Occup. Hyg. 45, 58595 (2001)

7. E.Van Deurssen, A. Pronk, S. Spaan, et al., Ann. Occup. Hyg. 58, 724-38 (2014)

8. C.B. Healy, M.A. Coggins, M. Van Tongeren et al., Ann. Occup. Hyg. 58, 6-18 (2014)

9. E. Monfort, M.J. Ibáñez, A. Escrig, et al., cfi/Ber. DKG 85, 36-42 (2008)

10. E. Monfort, M.J. Ibáñez, A. Escrig, In Safety, reliability and risk analysis: Theory, methods and applications. London: Taylor and Francis, pp. 274346 (2009)

11. E. Monfort, A. Mezquita, E. Vaquer et al., In Comprehensive Materials Processing. Bassim: Elsevier, 8, pp. 71-102, (2014)

12. C. Ziemann, O. Creutzenberg, M.J. Ibañez, et al., cfi/Ber. DKG 91，63-9 (2015)

13. European Trade Union Confederation (2007) Response to the 2nd phase consultation on Directive 2004/37/EC

14. International Agency for Research on Cancer (2012): IARC Monographs on the evaluation of the carcinogenic risk of chemicals to humans: A review of human carcinogens. Part C: arsenic, metals, fibres, and dusts. IARC Monographs, pp. 196-211, (2012)

15. H.W. Schlipköter, A. Brockhaus, J. Molec. Medicine, 39, 22, 1182-1189 (1961)

16. Castranova, V. (Eds.) Silica and Silica-Induced Lung Diseases. Boca Raton: CRC Press, pp. 293298 (1996)

17. Castranova, V.; van Dyke, K.; Wu, L.; Dalai, N.S.; Vallyathan, V. (Eds.) Silica and Silica-Induced Lung Diseases. Boca Raton: CRC Press, pp. 283291 (1996)

18. R. Duffin, P.S. Gilmour, R.P.F. Schins, A. Clouter, K. Guy, D.M. Brown, W. MacNee, P.J. Borm, K. Donaldson, V. Stone, Toxicol. App. Pharm. 176(1),10-17 (2001)

19. R. Bégin, S. Massé, M. Rola-Pleszczynski, M. Martel, Y. Desmarais, M. Geoffroy, L. Le Bouffant, H. Daniel, J. Martin, Exp. Lung Res. 10, 385-399 (1986)

20. G.M. Brown, K. Donaldson, D.M. Brown, Toxicol. App. Pharm. 101, 1, 95-105 (1989)

21. C. Albrecht, A.M. Knaapen, A. Becker, D. Höhr, P. Haberzettl, F.J. van Schooten, P.J.A. Borm, R.P.F. Schins, Resp. Res., 6, 129, (2005)

22. C. Ziemann, S.M. Reamon-Buettner, T. Tillmann, H.T. Hansen, M.J. Ibáñez, E. Monfort, G. Bonvicini, A. Escrig, O. Creutzenberg, In: Deutsche Gesellschaft für experimentelle und klinische Pharmakologie und Toxikologie (2014) 
23. R.P. Nolan, A.M. Langer, J.S. Harington, G. Oster, I.J. Selikoff, Environ. Res. 26, 2, 503-520, (1981)

24. C. Ziemann, A. Escrig, G. Bonvicini, M.J. Ibáñez, E. Monfort, A. Salomoni, O. Creutzenberg, Annals of Work Exposures and Health, 61, 4, 468-480 (2017) 